\title{
On the plasma sheet dependence on solar wind and substorms and its role in magnetosphere-ionosphere coupling
}

\author{
V A Sergeev ${ }^{1 *}$, N P Dmitrieva ${ }^{1}$, N A Stepanov ${ }^{1,2}$, D A Sormakov ${ }^{2}$, V Angelopoulos ${ }^{3}$ and A V Runov ${ }^{3}$
}

\begin{abstract}
Recently, it was argued that Hall conductivity and peak intensity of equivalent ionospheric currents are sensitive to the amount of field-aligned acceleration of plasma sheet (PS) electrons, which in turn depends on the plasma sheet parameters $T_{e}$ and $N_{e}$ (electron temperature and density) proportionally to the quantity eTN $=\left(T_{e}\right)^{1 / 2} / N_{e}$. Here we extend these studies using data from six tail seasons of THEMIS observations to show statistically that the behavior of these PS electron parameters, measured in the middle of the nightside plasma sheet at $\sim 10 R_{E}$ distance, depends in a very different way on two basic processes: the solar wind state and substorms. We confirm previous work that slow/dense (fast/tenuous) solar wind provides cold/dense (hot/tenuous) plasma sheet conditions. However, we find that electron temperature and pressure parameters $\left(T_{\mathrm{e}}\right.$ and $\left.P_{\mathrm{e}}\right)$ behave differently from the proton ones $\left(T_{\mathrm{p}}\right.$ and $\left.P_{\mathrm{p}}\right)$, indicating a strong decoupling between temperature variations of auroral protons and electrons in the central plasma sheet (CPS): electrons are more sensitive to the substorm-related acceleration in the magnetotail than protons. Our superposed epoch study of plasma sheet parameter variations during substorms as well as our analysis of plasma acceleration at dipolarization fronts shows that during the substorm expansion phase a new (accelerated and plasma-depleted) population comes into the inner CPS with the flow bursts, showing an average increase of electron temperature and eTN parameter roughly by a factor of 2 above its background values for both cold/dense and hot/tenuous plasma sheet states. Preferential electron heating in the flow bursts is also statistically confirmed.
\end{abstract}

Keywords: Plasma sheet; Particle acceleration; Solar wind driver; Substorms; Flow burst

\section{Findings Introduction}

Auroral zone magnetic perturbations and associated magnetic indices (especially, the auroral electrojet low (AL) index) are commonly used to characterize the intensity, timing, and types (or phases) of magnetospheric activity. Observationally, Newell and Gjerloev (2011) found a close correlation between the AL index and global auroral power (a measure of total precipitated energy flux) demonstrating that conductivity variations may effectively control the changes of AL index amplitude. How these conductivity variations respond to the variations of magnetospheric drivers (convection and

\footnotetext{
*Correspondence: victor@geo.phys.spbu.ru

${ }^{1}$ Earth Physics Department, St. Petersburg State University, Ulyanovskaya 1, Petrodvoretz, St. Petersburg 198504, Russia

Full list of author information is available at the end of the article
}

field-aligned currents) on the one hand and the solar wind driver on the other hand are still big open questions. In particular, since many different processes actually contribute to the ionospheric conductivity, how these processes conspire to control the conductivity and AL response remains to be understood. According to the Fukushima theorem (Fukushima 1976), in a relatively uniformly conducting ionosphere, the ground magnetic perturbation amplitude depends mainly on the ionospheric Hall conductivity, which-at the nightside-is caused by the precipitation of accelerated magnetospheric electrons.

Recently, Sergeev et al. (2014) (herein referred to as Paper 1) noted that the typical energy of magnetospheric electrons $\left(T_{\mathrm{e}}\right)$ varies between 0.2 and $1 \mathrm{keV}$ in the plasma sheet and is too low to affect Hall conductivity that is produced at the E-layer of the ionosphere since such energies

\section{Springer}

(c) 2016 Sergeev et al. Open Access This article is distributed under the terms of the Creative Commons Attribution 4.0 International License (http://creativecommons.org/licenses/by/4.0/), which permits unrestricted use, distribution, and reproduction in any medium, provided you give appropriate credit to the original author(s) and the source, provide a link to the Creative Commons license, and indicate if changes were made. 
cannot penetrate deep enough into E-layer ionospheric altitudes. The Hall conductivity should mostly respond to the accelerated, high-energy tail of the electron spectrum, as confirmed observationally by Ostgaard et al. (2002). Concerning the related acceleration mechanisms, fieldaligned electron acceleration in regions of strong upward field-aligned current (UFAC) is among the main candidates (Paschmann et al. 2003). Recently, its importance was experimentally confirmed by Korth et al. (2014) who demonstrated a global correspondence and intensity correlation between UFAC densities and auroral power on meso-scale grid size (hundreds of $\mathrm{km}$ ). In the case of large field-aligned electric potential drop, its amplitude is controlled by the parameter eTN $=\left(T_{\mathrm{e}}\right)^{1 / 2} / N_{\mathrm{e}}$ (Knight 1973; Paschmann et al. 2003); this parameter is an important state variable of the magnetotail. For the same level of magnetospheric drivers (field-aligned currents), high eTN values (as expected in a hot/tenuous plasma sheet) is capable of producing stronger electron acceleration and larger Hall conductivity compared to low eTN values (as in a cold/dense plasma sheet). This was recently confirmed in Paper 1 by demonstrating that the AL magnitude, when normalized to the dayside merging intensity $E_{\mathrm{kl}}$ (defined as $E_{\mathrm{kl}}=\mathrm{VB} \sin ^{2}(\theta / 2)$ ), indeed depends on eTN as directly evaluated in the tail plasma sheet. Therefore, it is now important to investigate the range of such variations and to understand the sources of the eTN variability that is so critical in allowing the plasma sheet to support strong field-aligned acceleration and strong Hall conductivity. We attempt to address these questions in this paper.

Paper 1 discussed two different processes affecting the eTN amplitude: One is the substorm-related plasma acceleration in the magnetotail, which provides accelerated and density-depleted (that is, high eTN) plasma. The second is the well-known dependence of plasma sheet density and temperature on solar wind (SW) parameters. We note, however, that most previous comparative studies dealt with proton parameters in the plasma sheet and solar wind, whereas systematic studies of electron parameters were rare. Motivated by the potential importance of plasma sheet parameters $\left(T_{\mathrm{e}}, N_{\mathrm{e}}, \mathrm{eTN}\right)$ for magnetosphere-ionosphere coupling, in this paper, we address in greater detail these controlling factors, namely, the influence of solar wind and substorms. Whereas most previous studies investigated the proton component (which is the major contributor to the plasma sheet pressure, e.g., Borovsky et al. (1998), Tsyganenko and Mukai (2003), and Wang et al. (2010)), here we emphasize the variations of the electron component in the nightside central plasma sheet (CPS) at distances 9-12 $R_{E}$. This plasma sheet region is quite important in the magnetosphere as a plasma source region for the inner magnetosphere, as it provides the seed population for the ring current and radiation belts. Also, this region maps into the middle of the auroral oval, where most bright auroras and peak magnetic perturbations are often observed. With regard to the solar wind driver, we address the hierarchy of controlling SW parameters and their cross-correlation time delays with plasma sheet parameters, emphasizing the difference in response between plasma sheet protons and electrons. With regard to substorm effects, we study the ensemble averaged plasma sheet response during substorms, under different initial states of the plasma sheet, by means of superposed epoch analysis. We also analyze the acceleration features related to the dipolarization fronts. One of our main goals will be to assess quantitatively the relative contributions of these two processes: solar wind state and substorms.

\section{Data analysis}

We investigate the database combining the 1-minaveraged central plasma sheet (CPS) parameters $(N, P$, and $T$, separately for protons and for electrons, and $\beta=$ $\left.2 \mu_{0}\left(P_{\mathrm{i}}+P_{\mathrm{e}}\right) / B^{2}\right)$. The measurements were selected from the nightside and dawn local time sectors (21 to 06 hours MLT) at distances between 9 and $12 R_{E}$ by the plasma spectrometer (ESA) covering energies between $5 \mathrm{eV}$ and $25 \mathrm{keV}$ for protons and electrons. We note that the proton temperature/pressure are somewhat underestimated during episodes of hot plasma sheet (when $T_{\mathrm{p}}$ exceeds, say, $10 \mathrm{keV}$ ) but that does not affect our results. The observations come from THEMIS-A, -D, and -E spacecraft near their apogees. At each minute, the data from one spacecraft, showing the largest $\beta$ value is selected, and only central plasma sheet $(\beta>1)$ samples are used in the following analyses. These data were complemented by 1min-averaged solar wind parameters $\left(N_{\mathrm{sw}}, V_{\mathrm{sw}}, T_{\mathrm{sw}}\right.$, and flow pressure $P_{\mathrm{d}}$ ) from the OMNI database, smoothed with a sliding 5-min window and jointed with the $\mathrm{AL}$ and $\mathrm{PC}$ index databases and solar-wind-based "dayside merging rate" $E_{\mathrm{kl}}=\mathrm{VB} \sin ^{2}(\theta / 2)$. This is the same database which was used in Paper 1, but it was extended for the purposes of the current analysis to include three more years (it now includes six THEMIS tail seasons between 2008 and 2013). One- and 3-h averages have been also formed and used when necessary.

Additionally, to obtain quantitative information about the plasma changes associated with individual flow bursts, we also used a different data set, consisting of THEMIS observations during 150 sharp magnetic fronts within flow bursts (otherwise known as dipolarization fronts (DFs)). This is a subset of a larger dataset consisting of thousands of DF events, previously identified during tail seasons 2008 to 2010 . Our subset includes only the events with the most significant changes in $B_{\mathrm{Z}}\left(\delta B_{\mathrm{Z}}>\right.$ $2 \mathrm{nT})$, recorded in the CPS $\left(\left|B_{\mathrm{x}}\right|<20 \mathrm{nT}\right.$ and $\left.\beta>1\right)$, at radial distances between 8.5 and $12 \mathrm{R}_{\mathrm{E}}$ in the nightside 
magnetotail. This is the same distance range used in the main data set. For this analysis, we included the data from the SST instrument on THEMIS that extends the energy range of the measurements well above $25 \mathrm{keV}$ (from $35 \mathrm{keV}$ to $1 \mathrm{MeV}$ ). The results are therefore accurate for electron and proton temperatures well above $10 \mathrm{keV}$.

\section{$A L$ effects of different plasma sheet states}

Figure 1 compares the 3-h-averaged AL amplitude and solar-wind-based $E_{\mathrm{kl}}$ average value for those 3-h-long intervals, in which $E_{\mathrm{kl}}$ variation and $\mathrm{PC}$ index variation (in the sunlit polar cap) are well correlated $(C C>0.5)$. The picture is very similar to Fig. 1 of Paper 1, although it is now obtained from twice the data volume but also using a strong restriction imposed on the plasma sheet coverage by THEMIS: we require that during the 3 -hlong time interval there should be $>50 \%$ plasma sheet measurement points in the high-beta plasma sheet, compared to more relaxed $20 \%$ requirement imposed in Paper 1. As usual, the ground magnetic variations (AL) correlate well with the dayside merging rate $\left(E_{\mathrm{kl}}\right)$, but the difference between different (eTN-based) plasma sheet states is also obvious in this plot. For large eTN ( $>3.5$, red points), the $E_{\mathrm{kl}}$ and $\mathrm{AL}$ values are well correlated $(\mathrm{CC}=-0.89)$ even though this data set has no points with large $E_{\mathrm{kl}}>2 \mathrm{mV} / \mathrm{m}$. For small eTN $(<1.5)$, the average slope is roughly $30 \%$ smaller; the correlation is also significant $(C C=-0.86)$ due to a number of points with large

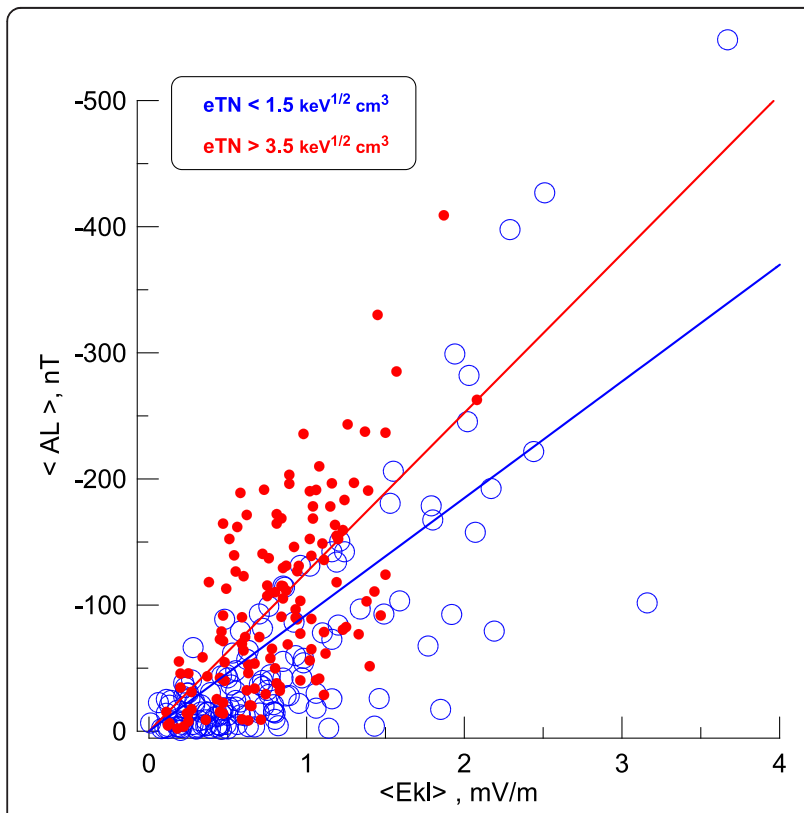

Fig. 1 Comparison of AL and $E_{k l}$ average values for 3-h-long intervals with correlated $E_{\mathrm{kl}}$ and $\mathrm{PC}$ index values (taken in the sunlit polar cap, CC $>0.5)$ and with good coverage of central plasma sheet by THEMIS (>50\% measurements with $\beta>1$ during each interval) driver intensity $\left(E_{\mathrm{kl}}>2 \mathrm{mV} / \mathrm{m}\right)$. According to Paper 1 , this difference is due to the different solar wind properties (this will also be confirmed later in this paper). The blue (low eTN) points occupy a broader sector, with many intervals showing low AL level $(<100 \mathrm{nT})$ even if the solar wind driver is strong $\left(<E_{\mathrm{kl}}>\sim 1-2 \mathrm{mV} / \mathrm{m}\right)$. These points demonstrate the effect of different solar wind state, as will be shown below. Here are also a number of high AL intensity events near the upper bound of the red point distribution. The latter points (as also will be shown below) demonstrate the effect of substorm activity.

\section{Plasma sheet-solar wind correlations}

The solar wind influences the plasma sheet parameters in a number of ways, each including different time constants or delays. An increasing solar wind flow pressure $P_{\mathrm{d}}$ quickly compresses the magnetotail, thereby immediately (within a few minutes) increasing the plasma sheet pressure (after taking into account the propagation time delays). Concerning the formation of a cold/dense plasma sheet in the tail center, double cusp reconnection under northern interplanetary magnetic field (IMF) conditions and subsequent slow penetration of mass-loaded, closed flux tubes from the flanks into the central tail sector is a much longer process, requiring as much as $\sim 8 \mathrm{~h}$ to transpire (Terasawa et al. 1997; Wang et al. 2010). Substormrelated effects are in-between these two timescales, as they have a delay about an hour to develop in the central plasma sheet (Borovsky et al. 1998). In view of this spread, we start from cross-correlation analysis of 1-h-averaged data to establish a hierarchy of solar wind controlling parameters. About 4500-5000 hourly data points from the same CPS region (9-12 $R_{E}, 21-06-h$ MLT) are available for the correlative study presented below.

Our result for proton pressure (top panel in Fig. 2) is consistent with the previous findings (Borovsky et al. 1998; Tsyganenko and Mukai 2003; Wang et al. 2010). It shows a high correlation $(C C \sim 0.7)$ with minimal time delays, with some (much smaller) influence of the solar wind electric field (see also Table 1). In a similar way, the CPS plasma density correlates well with the solar wind density, and the CPS temperature ( $T_{\mathrm{p}}$, not shown here) correlates best with the solar wind velocity and temperature. The effects of solar wind electric field are marginal for proton parameters. These CPS proton correlations with solar wind parameters are mostly known from previous studies.

Surprisingly, however, quite a different picture emerges for electron parameters. According to Fig. 2, bottom plot, the electron pressure is no longer fully controlled by the solar wind dynamic pressure: here the major control is assumed by the merging electric field $E_{\mathrm{kl}}$ which shows higher correlation with electron pressure during current and preceding hours. A slightly larger 


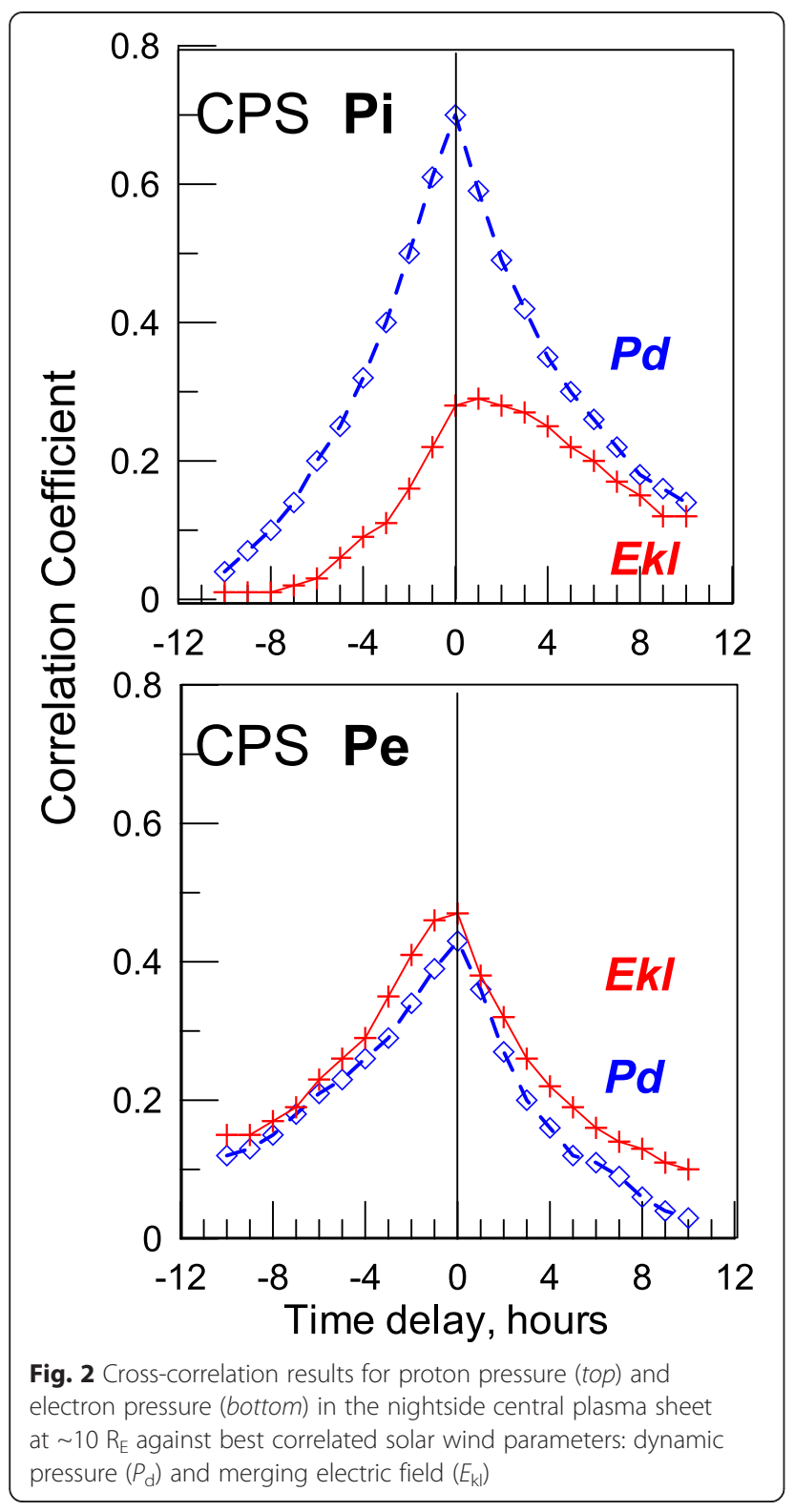

peak correlation is provided by the AL index (see Table 1, where the underlined solar wind quantities in the multiple regression fits are normalized to average values $N=5 \mathrm{~cm}^{-3}, T=10^{5} \mathrm{~K}, V_{\mathrm{sw}}=400 \mathrm{~km} / \mathrm{s}, E_{\mathrm{kl}}=1 \mathrm{mV} / \mathrm{m}$, and $\mathrm{AL}=-100 \mathrm{nT}$ ). These are major indicators of the increasing importance of substorm effects for the plasma sheet electron component, as compared to the proton component. The linear correlation coefficient between $P_{\mathrm{p}}$ and $P_{\mathrm{e}}$ is not large $(\mathrm{CC}=0.48$ for hourly means and 0.34 for high-beta $1 \mathrm{~min}$ values in our data set), illustrating a strong decoupling between the temperature variations of auroral protons and electrons in the CPS (whereas CC $=0.99$ between $N_{\mathrm{e}}$ and $N_{\mathrm{p}}$ in the CPS as dictated by the quasi-neutrality requirement).

\section{Substorm effects at $R \sim 11 R_{E}$}

To analyze statistically the effects of substorms on electron parameters in the CPS, we carried out a superposed epoch study of electron variations during isolated substorms. Based on the OMNI data, we selected the events with southward IMF turning which occurred on the quiet AL index background. Following the tradition, substorm onset ( $T=0$ time for the superposed epoch study) was determined as the onset of the sharp AL drop that proceeds to exceed $100 \mathrm{nT}$ in variation. Altogether, we identified 55 events for this analysis.

Figure 3 confirms the substorm-related electron heating ( $T_{\mathrm{e}}$ increase), density reduction, and overall increase of eTN following substorm onset, which is also consistent with the picture of nightside plasma injections activated during substorm. High/low eTN subsets are chosen according to the average value $<\mathrm{eTN}>$ during one full hour preceding the substorm onset. Namely, we identified 25 events starting under $<\mathrm{eTN}><1 \mathrm{keV}^{1 / 2} \mathrm{~cm}^{3}$ (cold/dense background, shown by blue color) and 12 events having $<\mathrm{eTN}>>2.5 \mathrm{keV}^{1 / 2} \mathrm{~cm}^{3}$ (hot/tenuous background, shown by red color). On average, the substorm-related increases of $T_{\mathrm{e}}$ and eTN are comparable in two data sets, both about a factor of 2 , and the separation of two curves is nearly the same both before and after the substorm. This also shows visually that eTN differences in the CPS produced by substorms (the relative changes in eTN) and those due to different solar wind-related background (the differences between the blue and red curves) are comparable.

As seen on the top plot, both data sets are characterized by a similar average solar wind driver ( $E_{\mathrm{kl}}$ amplitude, second panel), but the AL index amplitude is systematically

Table 1 Peak coefficients of paired correlation (CC) between 1-h-averaged central plasma sheet and solar wind (and AL index) parameters and best multiple regression fits

\begin{tabular}{|c|c|c|c|c|c|c|c|}
\hline $\mathrm{CC}$ & $P_{\mathrm{d}}$ & $N_{\text {sw }}$ & $T_{\mathrm{sw}}$ & $V_{\mathrm{sw}}$ & $|\mathrm{AL}|$ & $E_{\mathrm{kl}}$ & Peak multiple regression and $\mathrm{CC}$ \\
\hline $\mathrm{NePS}_{\mathrm{e}} \mathrm{cm}^{-3}$ & 0.57 & 0.62 & 0.05 & 0.2 & 0.12 & 0.13 & $N_{\mathrm{ePS}}=0.61 \underline{N}_{s w}^{0.54}, C C=0.63$ \\
\hline$P_{\mathrm{iPS}}, \mathrm{nPa}$ & 0.70 & 0.68 & 0.05 & 0.11 & 0.15 & 0.28 & $P_{\mathrm{iPS}}=0.06+0.16 \underline{N}_{s W} \underline{V}_{s W}^{2}+0.04 \underline{E}_{k \prime,} C C=0.76$ \\
\hline$P_{\text {ePs, }} \mathrm{nPa}$ & 0.42 & 0.25 & 0.24 & 0.16 & 0.49 & 0.46 & $P_{\mathrm{ePS}}=0.14\left(\underline{N}_{s W} \underline{V}_{s W}^{2}\right)^{0.39} \underline{E}_{k l}^{0.14} \underline{A L}^{0.15}, C C=0.64$ \\
\hline$T_{\mathrm{ePS}}, \mathrm{keV}$ & -0.04 & -0.23 & 0.25 & 0.30 & 0.44 & 0.32 & $T_{\mathrm{ePS}}=-0.09-0.13 \underline{\mathrm{N}}_{s W}+0.81 \underline{\mathrm{V}}_{s W}+1.18 \log \underline{A L}, C C=0.58$ \\
\hline $\mathrm{eTN}, \mathrm{keV}^{1 / 2} \mathrm{~cm}^{3}$ & -0.40 & -0.53 & 0.15 & 0.28 & 0.21 & 0.06 & $\mathrm{eTN}=2.93-2.05 \log \left(\underline{N}_{s w} \underline{V}_{s w}^{2}\right)+0.63 \underline{V}_{s W}+1.09 \log A L_{1}, C C=0.62$ \\
\hline
\end{tabular}

Bold values indicate the parameters showing highest correlation 

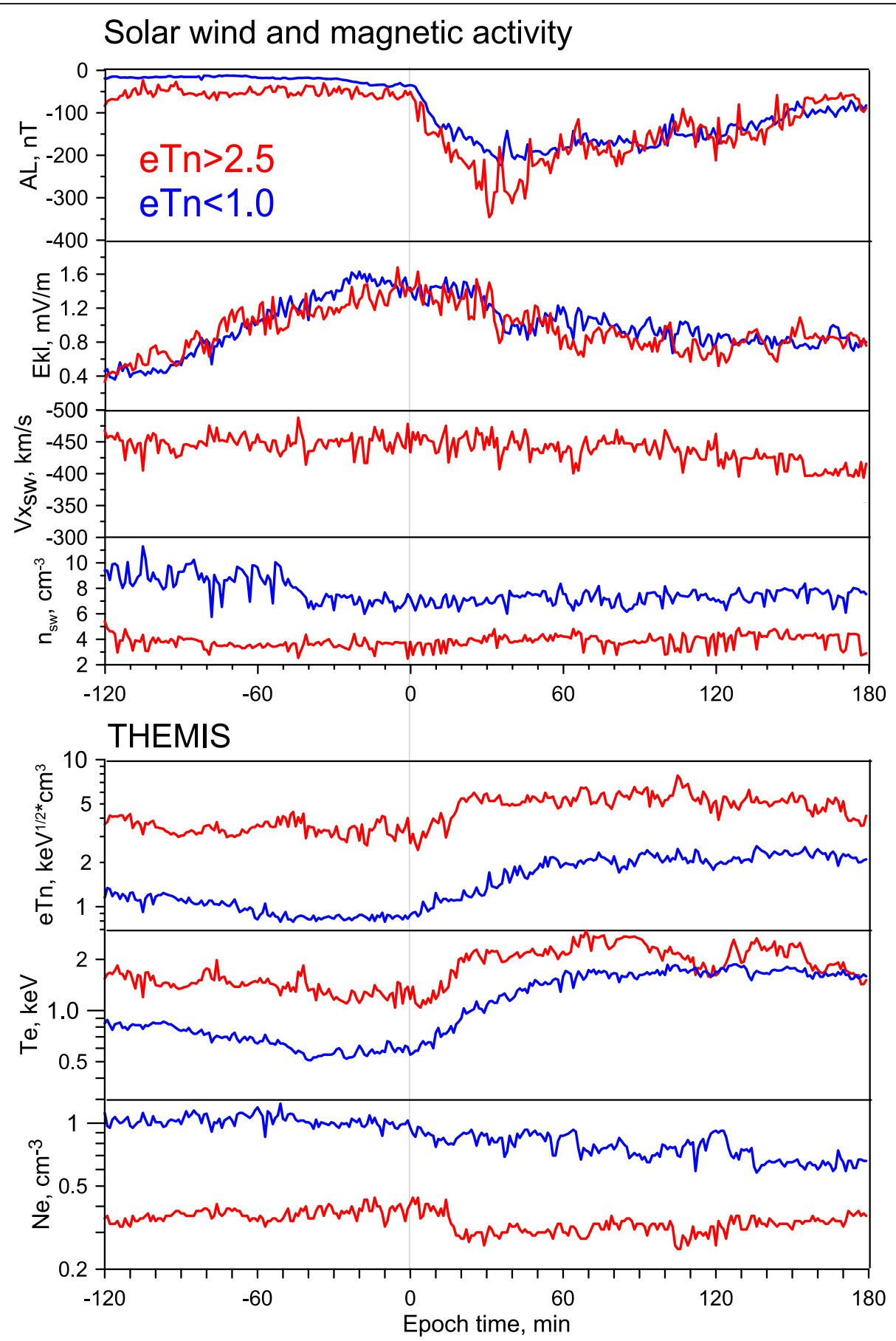

Fig. 3 Superposed epoch substorm behavior of the solar wind drivers and AL index (top) as well as CPS parameters at 9-12 RE in the nightside magnetosphere

stronger during high eTN case (first panel), consistent with the results of Fig. 1.

\section{Role of the flow bursts}

We now use the THEMIS observations of 150 sharp dipolarization fronts to study plasma sheet electron heating. The events are distributed in $Y$ between -5 and
$7 R_{E}$, and not all of them are entirely isolated, that is, there could be preceding activity in the plasma sheet. For all cases, data from ESA and SST are combined to include the high-energy particles in the moments derivation. Below, we concentrate on the changes occurring between the parameters preceding the DF (averaged over [ $t_{0}-2 \mathrm{~min}$ and $\left.t_{0}-1 \mathrm{~min}\right]$ time interval, where $t_{0}$ is the 
DF start time) and following after the DF (during $40 \mathrm{~s}$ after the DF peak).

Figure 4 illustrates the main findings. Both proton and electron temperatures tend to increase after entering the flow burst (points lying above the diagonal in Fig. 4a). Electrons on average gain more energy (on average, $T_{\mathrm{e}}$ increases by a factor of 1.7, Fig.4c) than the protons (on average, $T_{\mathrm{p}}$ increases by a factor of 1.4). Only electrons display occasionally temperature increases exceeding a factor of 3 (above the corresponding line in Fig. 4a). Due to such preferential electron heating, a population with $T_{\mathrm{p}} / T_{\mathrm{e}} \sim 1$ sometimes is seen in the right bottom corner of Fig. $4 \mathrm{~b}$, which is unusual in the magnetotail. The close correlation between the background temperature and post-DF plasma temperatures implies that the population coming with the flow burst forms from the background plasma (these two populations are not independent, like it could be in one of the options of the reconnection scenario in which fresh reconnection-accelerated plasma is coming in with a flow burst). In this part, results of this simple analyses support the main conclusions of superposed epoch analyses that (1) the background parameters of the plasma sheet strongly influence the resulting post-substorm values and that (2) the substorm-related eTN increase is about a factor of 2 independent of the initial state (average eTN increase at the DF is about a factor of 1.7 in Fig.4c).

\section{Discussion}

Our study provides the quantitative estimates of the electron CPS parameter variations in the region between 9 and $12 R_{E}$ on the nightside, which are caused by two different factors: the solar wind state and substorms.

The solar wind state controls the background values of many parameters, with the CPS density (CPS proton pressure) being primarily regulated by the SW density (SW flow pressure), consistent with Borovsky et al. (1998), Tsyganenko and Mukai (2003), and many similar previous studies. The time constants in these processes vary from several minutes (for pressure response) to several hours (for density response, to form the cold/dense plasma sheet, e.g., Terasawa et al. (1997), Wang et al. (2010)). In past studies, information about the behavior of the electron pressure and temperature was scarce, and the controlling factors for these parameters were still not obvious. Changing the previous opinion that $T_{\mathrm{p}} / T_{\mathrm{e}}$ ratio is more/less stable and large, about $\sim 7$ (e.g., Baumjohann (1993)), Artemyev et al. (2011) showed that, except for rare cold sheets, the average ratio is smaller ( 3.5). Also, Wang et al. (2012) showed large variations $T_{\mathrm{p}} / T_{\mathrm{e}}$ in the plasma sheet, particularly, its decrease in association with large solar wind velocity and strong SW electric field (southward IMF) presumably also correlated to substorms. Luo et al. (2011) investigated the factors controlling the high-energy ( $>38 \mathrm{keV}$ ) electron flux and also reported on its basic dependence on $V_{\mathrm{sw}}$ and southward IMF preceding the observations by $1-1.5 \mathrm{~h}$. Our results complement these studies by showing (a) weak correlation between proton and electron pressures $(\mathrm{CC}=0.48)$ in the CPS and $(\mathrm{b})$ the dominant effect of substorms on both $T_{\mathrm{e}}$ and $P_{\mathrm{e}}$; statistically, the best correlation is provided by the $E_{\mathrm{kl}}$ and AL parameters (Table 1). The strong decoupling between the temperature variations of protons and electrons in the central plasma sheet (presumably also reflected in the auroral spectra) is an unexpected and interesting new feature which requires a further investigation.

Further information that helps address the reasons for the differences between electron and proton acceleration

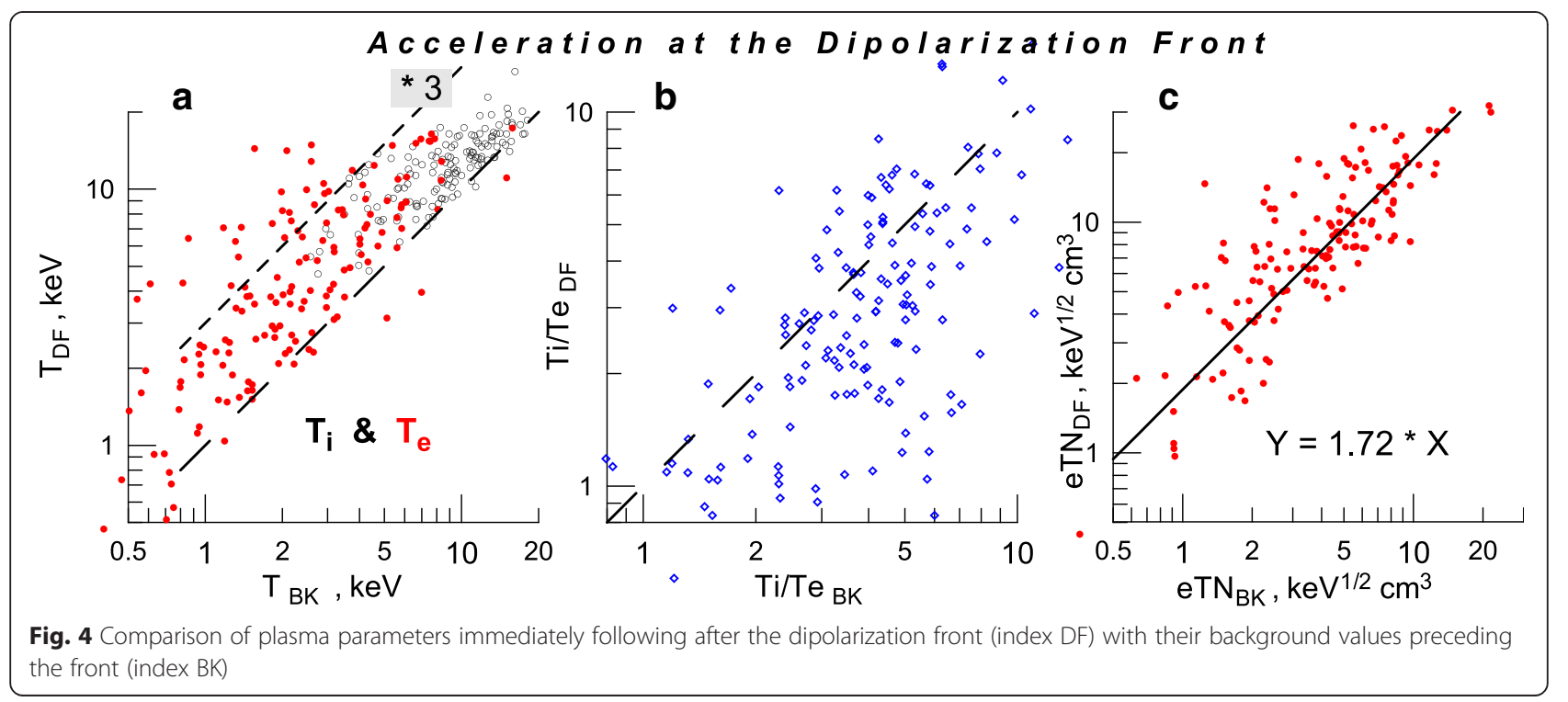


in the plasma sheet comes from our analyses of acceleration at the dipolarization fronts. It shows that electrons on average gain more energy than the protons and that only electrons display occasionally a large temperature increase, exceeding a factor of 3 . Preferential acceleration of electrons in the flow bursts (or at the DFs) is thus an observational fact. This interesting problem is outside the scope of our brief paper.

All parts of our study confirm that two different factors, solar wind state and substorm acceleration, provide comparable contributions to the variations of $T_{\mathrm{e}}$, $P_{\mathrm{e}}$, and eTN. In the statistical SW correlative study, this is manifested as generally low paired correlation coefficient values $(\mathrm{CC}<\sim 0.5)$ with individual solar wind parameters, showing comparable statistical contributions of dayside merging electric field $E_{\mathrm{kl}}$ and solar wind dynamic pressure. In the substorm epoch study (Fig. 3), we obtained a roughly twofold eTN and $T_{\mathrm{e}}$ increase for both cold/dense and hot/tenuous populations. A similar twofold $T_{\mathrm{e}}$ and eTN increase, on average, is observed at the dipolarization fronts, confirming the appearance of newly heated plasma, energized within the BBFs by the arrival of dipolarized flux bundles from the tail, as the main mechanism of $T_{\mathrm{e}}$ and eTN variations during substorms. In the most visual form, the comparable strength of two processes (substorms and solar wind state) is seen in the scatter plots Fig. 4a, c, which show that the $T_{\mathrm{e}}$ and eTN values after the acceleration are still mostly affected by its pre-DF value. The fact that plasma in the incoming flow burst is only modestly heated was also noted by Gabrielse et al. (2014) and Runov et al. (2015) papers.

The problem of preferential electron acceleration in the flow bursts is a rather new puzzle. There are different reasons providing asymmetry between two species. One of them is, certainly, the difference in mass and gyroradius values, which make the particle motion in the current sheet and flow burst non-adiabatic (adiabatic) for protons (electrons), correspondingly. Moreover, Wang et al. (2012) showed that a simple twofluid description (with Maxwellian protons and electrons) of plasma sheet population is not supported by the observed particle spectra, which often can rather be represented as a mixture of two (cold and hot) populations. We suggest that such a two-component appearance may reflect two different processes forming the plasma sheet. Its particles are mostly provided by the (cool) solar wind source, coming into the central tail sector by means of interchange/diffusion process (Wang et al. 2010), where some particles succeed to be accelerated in spatially limited reconnection-produced fast flow channels. Energy-dependent magnetic drifts together with the ionospheric source of cold plasma add more complexity to that picture, which certainly requires a special study.

\section{Conclusions}

We analyzed the multi-year dataset of THEMIS at 9-12 $R_{E}$ in the nightside plasma sheet combined with the OMNI data. Our results show that parameters of the electron CPS in the magnetosphere, which control the efficiency of field-aligned acceleration $\left(T_{\mathrm{e}}\right.$ and $\left.\mathrm{eTN}\right)$, vary in a wide range due to the combined action of two different factors, solar wind state and substorms. We find that the main modification of the electron plasma (arrival of heated/depleted plasma causing average increase of $T_{\mathrm{e}}$ and eTN by a factor of 2) is provided by the flow bursts coming from the tail during substorms. We also find that the plasma sheet electron component is more prone to heating during substorms: it often shows a larger relative energy increase compared to the protons, which are more sensitive to the solar wind state. The plasma sheet ability to support the field-aligned acceleration, therefore, is varied in a wide range by the combined action of solar wind state and substorms, which have different dynamics and provide comparable partial contributions to these variations.

\section{Competing interests}

The authors declare that they have no competing interests.

\section{Authors' contributions}

All authors read and approved the final manuscript.

\section{Acknowledgements}

We thank the data providers, NSSDC at NASA GSFC for the OMNI data, Kyoto WDC for the AL index data, and C. W. Carlson and J. P. McFadden for the use of the ESA THEMIS data. This work was supported by the Russian Foundation of Basic Research grant 13-05-00132.

\section{Author details}

${ }^{1}$ Earth Physics Department, St. Petersburg State University, Ulyanovskaya 1, Petrodvoretz, St. Petersburg 198504, Russia. ${ }^{2}$ Arctic and Antarctic Research Institute, St. Petersburg, Russia. ${ }^{3}$ University of California, Los Angeles, CA, USA.

Received: 26 March 2015 Accepted: 24 July 2015

Published online: 19 August 2015

\section{References}

Artemyev AV, Baumjohann W, Petrukovich AA, Nakamura R, Dandouras I, Fazakerley A (2011) Proton/electron temperature ratio in the magnetotail. Ann Geophys 29(12):2253-2257. doi:10.5194/angeo-29-2253-2011

Baumjohann W (1993) The near-Earth plasma sheet: an AMPTE/IRM perspective. Space Sci Rev 64(1-2):141-163. doi:10.1007/BF00819660

Borovsky JE, Thomsen MF, Elphic RC, Cayton TE, McComas DJ (1998) The transport of plasma sheet material from the distant tail to geosynchronous orbit. J Geophys Res 103(A9):20297-20331

Fukushima N (1976) Generalized theorem for no ground magnetic effect of vertical currents connected with Pedersen currents in the uniformconductivity ionosphere. Rep lonos Space Res Jap 30:35-50

Gabrielse C, Angelopoulos V, Runov A, Turner DL (2014), Statistical characteristics of particle injections throughout the equatorial magnetotail, J. Geophys. Res. Space Physics, 119, 2512-2535. doi:10.1002/2013JA019638.

Knight S (1973) Parallel electric fields. Planet Space Sci 21:741-750

Korth H, Zhang Y, Anderson BJ, Sotirelis T, Waters CL (2014) Statistical relationship between large-scale upward field-aligned currents and electron precipitation. J Geophys Res Space Physics 119:6715-6731. doi:10.1002/2014JA019961

Luo B, Tu W, Li X, Gong J, Liu S, Burin Des Roziers E, Baker DN (2011) On energetic electrons ( $>38 \mathrm{keV}$ ) in the central plasma sheet: data analysis and modeling. J Geophys Res 116:A09220. doi:10.1029/2011JA016562 
Newell PT, Gjerloev JW (2011) Evaluation of SuperMAG auroral electrojet indices as indicators of substorms and auroral power. J Geophys Res 116:A12211. doi:10.1029/2011JA016779

Ostgaard N, Vondrak RR, Gjerloev JW, Germany G (2002) A relation between the energy deposition by electron precipitation and geomagnetic indices during substorms. J Geophys Res 107(A9):1246. doi:10.1029/2001JA002003

Paschmann G, Haaland S, Treumann R (eds) (2003) Auroral Plasma Physics, Space Sci. Series of ISSI, vol 15. Kluwer Acad. Publ, Dordrecht, Also Space Sci. Revs, 103, N1-4, 2002

Runov A., V. Angelopoulos, C.Gabrielse, J. Liu, D.L. Turner, X.-Z. Zhou (2015), Average thermodynamic and spectral properties of plasma in and around dipolarizing flux bundles, J.Geophys.Res., 120, N6, 4369-4383. doi:10.1002/ 2015JA021166

Sergeev, V. A., D. A. Sormakov, V. Angelopoulos (2014) A missing variable in solar wind-magnetosphere-ionosphere coupling studies. Geophys. Res. Lett. 41. doi:10.1002/2014GL062271

Tsyganenko NA and Mukai T (2003), Tail plasma sheet models derived from Geotail particle data, J. Geophys. Res., 108(A3), 1136. doi:10.1029/ 2002JA009707

Terasawa T et al (1997) Solar wind control of density and temperature in the near-Earth plasma sheet: WIND/GEOTAIL collaboration. Geophys Res Lett 24:935-938. doi:10.1029/96GL04018

Wang C-P, Lyons LR, Nagai T, Weygand JM, Lui ATY (2010) Evolution of plasma sheet particle content under different inter-planetary magnetic field conditions. J Geophys Res 115:A06210. doi:10.1029/2009JA015028

Wang C-P, Gkioulidou M, Lyons LR, Angelopoulos V (2012) Spatial distributions of the ion to electron temperature ratio in the magnetosheath and plasma sheet. J Geophys Res 117:A08215. doi:10.1029/2012JA017658

\section{Submit your manuscript to a SpringerOpen ${ }^{\circ}$ journal and benefit from:}

- Convenient online submission

- Rigorous peer review

- Immediate publication on acceptance

- Open access: articles freely available online

- High visibility within the field

- Retaining the copyright to your article

Submit your next manuscript at $\gg$ springeropen.com 